http://dx.doi.org/10.35381/racji.v5i9.730

\title{
La vida digna en el proceso de muerte y la eutanasia
}

Dignified life in the process of death, and euthanasia

Walter Ruperto Mogrovejo-Lituma

walter.mogrovejo@psg.ucacue.edu.ec

Universidad Católica de Cuenca, Cuenca

Ecuador

https://orcid.org/0000-0003-2392-6116

Juan Carlos Erazo-Álvarez

jcerazo@ucacue.edu.ec

Universidad Católica de Cuenca, Cuenca

Ecuador

https://orcid.org/0000-0001-6480-2270

Cecilia Ivonne Narváez-Zurita inarvaez@ucacue.edu.ec

Universidad Católica de Cuenca, Cuenca

Ecuador

https://orcid.org/0000-0002-7437-9880

José Luis Vázquez-Calle

jlvazquezc@ucacue.edu.ec

Universidad Católica de Cuenca, Cuenca

Ecuador

https://orcid.org/0000-0003-4980-6403

Recibido: 13 de abril de 2020

Revisado: 20 de abril de 2020

Aprobado: 24 de mayo de 2020

Publicado: 08 de junio de 2020 


\title{
RESUMEN
}

La vida digna en el proceso de muerte, supone un derecho que todos deben poseer, el acto de morir es un acto humano. La eutanasia deriva de los vocablos griegos eu=bueno $y$ thanatos = muerte que significan buena muerte, deben existir alternativas que eviten el sufrimiento, ofreciendo principios de autonomía y libertad de los seres humanos que se enfrentan al final de la vida. El objetivo es cómo reconocer la eutanasia como derecho constitucional en el Ecuador. La investigación fue de tipo no experimental y mixta. Como resultados se evidencia que la Constitución de la República del Ecuador, reconoce el derecho a una vida digna por lo tanto estaría reconociendo todas las etapas de esta vida hasta la muerte. La Corte Constitucional debería realizar una interpretación evolutiva como lo hizo con la sentencia del matrimonio igualitario, y así se podría reconocer a la eutanasia como un derecho.

Descriptores: Eutanasia; derechos civiles, derechos humanos; enfermedad. (Palabras Tomadas del Tesauro UNESCO).

\begin{abstract}
A dignified life in the process of death supposes a right that everyone must possess, the act of dying is a human act. Euthanasia derives from the Greek words eu = good and thanatos = death that mean good death, there must be alternatives that avoid suffering, offering principles of autonomy and freedom for human beings who face the end of life. The objective is how to recognize euthanasia as a constitutional right in Ecuador. The research was non-experimental and mixed. As results, it is evident that the Constitution of the Republic of Ecuador recognizes the right to a dignified life, therefore, it would be recognizing all stages of this life until death. The Constitutional Court should make an evolutionary interpretation as it did with the sentence of equal marriage, and thus euthanasia could be recognized as a right.
\end{abstract}

Descriptors: Euthanasia; civil and political rights; human rights; diseases. (Words taken from the UNESCO Thesaurus).

\section{INTRODUCCIÓN}

En los últimos años la medicina y la tecnología han evolucionado de manera positiva, mismas que han cumplido un objetivo importante en los seres humanos; esto refiere a la prolongación de la vida; sin embargo, en ciertos casos de personas con problemas graves de salud o en fases terminales de enfermedades catastróficas o incurables, se estaría dando una prolongación de sufrimiento y agonía; por lo tanto, estos avances no deberían 
ser utilizados solo para tratar de curar, dejando de lado el enfoque biomédico e incluir el enfoque psicológico y social, en el cuál estaría inmersa la legislación de cada país para buscar avances en las prácticas médicas, contemplando planos espirituales, legales y sentimentales dentro de la noción de calidad de vida; evitando el sufrimiento del ser humano en agonía, tratando de aplicar un ámbito, ético, médico y jurídico, englobando términos como la vida digna y la eutanasia.

Con base en lo manifestado, existen varios derechos regulados en nuestra constitución, entre los que se encuentra contemplada la vida digna; $y$, se encuentra codificado con un sinnúmero de condiciones y preceptos que permiten a los habitantes del territorio ecuatoriano sobrellevar su existencia de una forma adecuada; al mismo tiempo, se encuentran enumeradas varias formas en las que el estado protegerá la vida de sus habitantes; como servicios básicos, actividades de ocio, actividades laborales, salud gratuita y alimentación entre otras.

En este contexto, los legisladores no se han enfocado en dar un valor especial al derecho de la "vida digna"; ya que, dentro de este tema no se ha considerado la muerte asistida o eutanasia; la cual, implica terminar con la existencia de una persona que no puede decidir por sí misma encontrándose en el final de la vida; o, con una complicación muy grave de su salud; y, a causa de ello, aplicar una forma más humanizada, misma que ha sido previamente consentida por el enfermo terminal o sus familiares (Lorda \& Barrio, 2012); esta petición del paciente puede canalizarse de dos formas diferentes, detalladamente reguladas en la Ley; una es mediante la petición directa al médico; y, otra mediante la Voluntad Anticipada.

En esta perspectiva, la constitución de la república, intenta preservar la vida de sus habitantes aplicando principios de vida digna; por lo tanto, la persona se podrá desarrollar conforme a sus valores y creencias; así también, el proceso de morir debería gozar de estas características y condiciones. La vida y la muerte son dos cosas muy diferentes, la primera es el inicio de todo, en que se incluyen varios derechos desde el primer día de nacido; y, la muerte es el fin de todo, la terminación de todos los derechos adquiridos al momento de nacer; por lo tanto, la vida digna debería amparar y beneficiar a los enfermos 
de gravedad; condicionalmente, por lo analizado en la legislación no se ha procurado regular esta parte tan importante en el final de la vida.

A su vez, las personas que se encuentran en fases terminales; $y$, no tienen capacidad de desarrollarse en una sociedad de forma activa e imposibilitados para realizar actividades que en su vida cotidiana realizaban; y, dependen de otras personas perdiendo así su autonomía en cuanto a movilidad y toma de decisiones, lo cual limita el goce de una vida digna.

Cabe señalar que, en la constitución ecuatoriana, la vida digna se preocupa solo por el desarrollo de las personas durante la misma, dejando de lado el legislar la terminación de la misma dignamente y por su propia voluntad (eutanasia), principalmente en cuanto esto implica procedimientos médicos y técnicos, mismos que evitan el sufrimiento del paciente, evitando que continúe con su vida en las graves condiciones en la que vive.

De este modo, en Ecuador se estaría vulnerando el derecho a la vida digna de personas en estado terminal; por lo que, sería fundamental que se trate este tema; y, aún más, sobre los conceptos de la vida digna en el proceso de la muerte y la eutanasia.

Por lo tanto, la problemática se resume en ¿Cómo reconocer la eutanasia como derecho constitucional en el Ecuador?; y como objetivo: proponer el reconocimiento del derecho a la eutanasia y a una muerte digna a partir de una interpretación por parte de la Corte Constitucional del Ecuador en beneficio de todas las personas que sufren de enfermedades en estados terminales; 0 , de enfermedades que no poseen una cura.

\section{Referencial teórico}

\section{La vida digna en el proceso de muerte para su reconocimiento constitucional}

La Constitución del Ecuador de 2008 reconoce el derecho a la vida digna en el artículo 66 numeral 2. Martínez-Sempere (2000) "la vida humana es algo más que mera existencia biológica. Debe ser digna para que se considere humana"(p.16) Señala también, que la dignidad de las personas refiere a la posibilidad de decidir cómo quiere vivir, o morir. La vida digna no se refiere únicamente a condiciones respecto a la buena manera de vivir una persona en una comunidad; o, lo que provee el estado, como 
alimento vestido, vivienda, agua, educación, trabajo, sino también a otras formas que están apegadas existencia como también su muerte (Martínez-Sempere, 2000).

Sobre la vida digna Amaro-Cano (1998) concluye, "con estos pacientes moribundos el problema debería radicar en optar por la calidad y no por la duración de la vida a partir del respeto a los deseos del paciente".

La vida digna y el respeto ante la misma, implica valorar la vida, no solo cuando ésta deja de serlo y se convierte en muerte; siendo así, no estaría gozando de esa calidad. Si existiese la posibilidad de elegir cuándo y cómo morir, manifestando el derecho a la vida digna y a una muerte digna; a través del derecho al libre desarrollo de la personalidad, tal como es reconocido por la Constitución del Ecuador en su artículo 66 numeral 5 "El derecho al libre desarrollo de la personalidad, sin más limitaciones que los derechos de los demás" (Asamblea Nacional, 2008).

Con respecto al primer punto, se debe analizar si se está respetando el derecho a la autonomía moral de las personas; $y$, su libertad de elección en estos casos. La respuesta sería muy obvia; ya que, existe un derecho que no debe ser vulnerado de ninguna forma, por el motivo de que una persona que se encuentra en un estado grave de salud está pidiendo ayuda para acabar con este sufrimiento.

Llegados a este punto, la vida digna requiere una muerte digna, todos los seres humanos anhelan una vida digna, deseando ello para sus familiares y también para el personal profesional que realiza estudios en búsqueda de mejores condiciones de vida.

Entonces, se debe aplicar lo que anhelamos en la búsqueda de mecanismos y una normativa correcta para que las personas que lo necesiten puedan acceder a una muerte digna, ya que morir dignamente se constituye en un derecho, en el que debe respetarse el deseo y las decisiones tomadas por cada persona (Arévalo-Arévalo, Erazo-Álvarez, Ormaza-Ávila, \& Narváez-Zurita, 2020).

Así que, una de las formas de cumplir este derecho a la vida digna es a través del reconocimiento de la facultad del enfermo a elegir en determinados casos, procedimientos tales como la eutanasia, cuando sus condiciones de vida le impiden desarrollarse de una forma adecuada. La eutanasia debe ser vista Constitucionalmente, 
en vista de que abarca derechos fundamentales como la vida, la integridad física, los principios de la dignidad humana, y el libre desarrollo de la personalidad, la libertad, la libertad ideológica y religiosa, el hecho de que no esté constitucionalizada no significa que ésta no deba ser estudiada en el ámbito constitucional (Alonso-Álamo, 2008). En ese mismo contexto, se denomina a la muerte digna como ortotanasia; en la cual, se incluye como un rechazo a los tratamientos que intenten prolongar la etapa de la muerte.

Por lo que es necesario, qué para los pacientes atravesando una enfermedad terminal, incurable; o, que les impida cumplir sus actividades de forma independiente, no se apliquen tratamientos ni cuidados paliativos; $y$, a su vez se pueda recurrir a una medida razonable, ésta solicitada por el paciente para que así llegue la muerte (Ludwing-Schmidt, 2013). Este concepto es muy acertado; por cuanto, si se posee una vida digna se debe culminar en una muerte digna, sin tratamientos que alarguen la misma, esto se ve como algo innecesario; dado que, de una u otra forma llegarán a morir y estos tratamientos se están administrando sin ninguna necesidad o fin y se está prolongando el sufrimiento del paciente.

A su vez, se observa, que hay ciudadanos que dedican su tiempo a cuidados en varios ámbitos, como en el educativo, salud y público; así como también, dedican su tiempo de forma voluntaria a velar por mejorar la condición de vida de los demás sin recibir nada a cambio; por lo tanto, esto es un buen punto de arranque para poder dedicar tiempo y tareas similares enfocadas al derecho de una muerte digna sin objeciones y que la misma sea considerada como un derecho (Montón, 2015). Nuestra especie ha buscado siempre una vida digna en todos los sentidos, intentando satisfacer sus necesidades en varios aspectos, lo que realmente es necesario para su subsistencia; como puede ser, un hogar, trabajo y salario dignos; y, así también una profesión que sea digna; con estos aspectos, se puede decir que la humanidad siempre busca lo mejor para tener una vida completamente digna; y así, poder exigir a un estado, respetar sus decisiones respecto a ésta, que es garantizada en la Carta Magna. Además, que si nuestra vida es digna porque no se podría exigir una muerte digna. 
Segun Amaro-Cano,(1998) "alguien que haya logrado una vida digna, es decir, una vida respetable y respetada, aspira igualmente a la dignidad de la muerte" (p.823). En la perspectiva que aquí se adapta, la vida digna es el camino a una muerte digna; y, sobre todo respetando su voluntad configurándose en una muerte digna. Por lo tanto, para todos los seres humanos mortales, no está en nuestras manos evitar que la muerte ocurra; sin embargo, lo que sí se puede hacer es impedir la auto eliminación o suicidio, cuidado a enfermos terminales con técnicas y prácticas.

Por lo que, al hablar de vida digna o muerte digna, se puede decir que cada persona debe tener autonomía propia para decidir la forma en la que quiere vivir o cómo quiere morir; ya que, esto solo sería una manifestación de la voluntad de la persona, que desea o no seguir viviendo. Se dice que no existe un derecho a morir; ya que, la existencia o vida de una persona es parte de la condición humana (Martínez-Sempere, 2000). No obstante, se han podido comprobar varios métodos para alargar o prolongar la vida en etapas terminales; dado que, existen mecanismos para que la vida siga de una forma artificial.

En los últimos cincuenta años se ha permitido prolongar la vida en forma artificial por lo cual esto se ha estado causando un alargue respecto a la agonía y sufrimiento del paciente ya que mediante la tecnología se ha proporcionado nuevos elementos los cuales han creado circunstancias que hacen necesario precisar conceptos respecto a la persona, muerte, el morir y calidad y dignidad de la vida y la muerte" (Escobar-Triana, 1998, p. 1-2).

Hecha esta salvedad, en la actualidad se puede prolongar la vida y agonía de un paciente en estado terminal; en la mayoría de casos, por petición de sus familiares, los mismos que tienen una esperanza de recuperación de su familiar querido; sin tomar en cuenta que están alargando el sufrimiento del paciente y suyo mismo; por lo tanto, la tecnología y la evolución en las prácticas médicas pueden ser muy beneficiosas; sin embargo, en estos casos no hay voluntad de una persona en una etapa como esta; debido a ello, sería adecuado analizar todos estos parámetros, para que cuando el paciente esté consciente antes de llegar a esta etapa, pueda decidir sobre vivir o morir; y así, no alargar su sufrimiento en la agonía.

Además, las personas que se encuentran en estadios avanzados de la enfermedad manifiestan algunos síntomas, mismos que afectan su estado anímico y prevalece el 
dolor físico acompañado de varios síntomas como: falta de aire o respiración, agitación, falta de apetito, vómito, sangrados, parestesias y alteraciones tanto en orina y como en deposición, las cuales causan sufrimiento al paciente el mismo que por su estado se encuentra al límite de su tolerancia debido a la gravedad de su enfermedad; por lo tanto, este sufrimiento no solo afecta a la persona que se encuentra enferma sino también a las personas que realizan el cuidado de los mismos los cuales deben hacer un gran esfuerzo físico y emocional para el cuidado de los mismos, sea bien la familia o el personal sanitario (Sarmiento-Medina, 2012). Viéndose toda esa sintomatología agravada por los efectos adversos de la medicación que reciben, empeorando su calidad de vida.

Cabe señalar, que este tema es muy controversial, como lo menciona Medina-Morales (2013) "estas técnicas requieren la participación de terceras personas ajenas al paciente y, la mayor parte de las veces, demandan la intervención de un profesional médico" (p.400). Por lo tanto, implica que todo un equipo, entre médicos y personas ajenas participan en el cuidado de esta persona enferma. Vida digna podemos decir que es un derecho innato a la persona desde el momento en que esta nace, mismo que se considera muy importante, porque abarca varios derechos como son: la vida, salud, educación y libertad, todos estos derechos, que son y pertenecen a la dignidad humana; esencialmente inviolables por sus características, y se deben respetar; tomando en cuenta que el Estado está en la obligación de garantizar una vida digna y buena para sus habitantes; así como una muerte en condiciones de dignidad.

El verdadero derecho, reside en la calidad mínima de esta vida; lo que ha dado en denominarse vida digna; o, vida con un mínimo exigible de dignidad. Lo que se traduce en la necesidad de posibilitar a toda persona un conjunto de garantías que permitan el despliegue de nuestras capacidades humanas; a manera de ejemplo, no se consideraría vida digna aquella vivida en esclavitud; pues, la persona no gozaría de la capacidad de determinar su ideal de vida plena; y éste, le sería impuesto contra su voluntad, Decesare (2004) "Tampoco constituirá vida digna la que soporta una persona carente de un mínimo de alimentos que le permita desarrollarse psicoafectivamente" (p.151). 
Como se señala, la vida digna tiene un sinfín de características que enfocan a varios derechos inherentes a las personas, que habitan en un estado de derecho, en el cual se respetan sus derechos y libertades; ya que, si una persona no está en goce y disfrute de sus derechos, no estaría viviendo una vida digna en su plenitud; por lo tanto, el estado es y será el único responsable en que esta vida digna y dignidad humana que caracteriza los habitantes de una nación, sea respetada así como también sus derechos.

\section{La dignidad y vida digna en la Constitución y la ley.}

Según la Asamblea Nacional (2008) en el art. 66 de nuestra Carta Magna Refiere a los derechos de libertad; y, en el numeral 2, reconoce y garantiza "el derecho a la vida digna

"que asegure la salud, alimentación y nutrición, agua potable, vivienda, saneamiento ambiental, educación, trabajo, empleo, descanso y ocio, cultura física, vestido, seguridad social y otros servicios sociales necesarios".

El artículo 37 del mismo cuerpo legal, establece que el Estado garantiza varias condiciones sobre el aseguramiento a personas adultas mayores, en específico el numeral 7 , mismo que hace referencia al acceso a una vivienda y asegura una vida digna. Como se puede apreciar, La constitución del Ecuador se enfoca específicamente a derechos sociales y económicos; $y$, no se puede apreciar derechos que se dirijan específicamente a lo que refiere una vida digna, o dignidad humana y salud; ya que, estos son los principales elementos de nuestro objeto de estudio en el presente caso.

El Plan Nacional de Desarrollo 2017-2021 Toda una Vida hace un amplio tratamiento de la vida digna. El objetivo 1 del mencionado plan, busca garantizar una vida digna con iguales oportunidades para todas las personas en este plan, se manifiniesta que el estado es el unico responsable para garantizar varios objetivos relacionados con la vida digna de todos sus habitantes. De igual modo, se dice que el derecho a la salud debe orientarse de manera especial hacia grupos de atención prioritaria y vulnerable. Este plan nacional de desarrollo pretende conseguir lograr una vida digna para todas las personas, en especial para aquellas en situación de vulnerabilidad, incluye la promoción de un 
desarrollo inclusivo que empodere a las personas durante todo su ciclo de vida (Secretaría Nacional de Planificación y Desarrollo, 2017).

Como se puede apreciar, la Constitución; y, la ley del plan nacional de desarrollo con respecto a la vida digna; intentan decir, que nuestro estado se encuentra en la obligación con sus habitantes para preservar ésta; pero se estaria quedando corta esta concepción; ya que, la Constitución y la ley, no ven mas alla de la vida digna, dignidad humana y el binestar de sus habitantes.

El artículo 55 "las medidas administrativas inmediatas de protección serán otorgadas seguidamente por las Juntas Cantonales de Protección de Derechos y las Tenencias Políticas cuando esté siendo vulnerado o se ha vulnerado el derecho a la integridad personal y la vida digna de la mujer". De igual manera, el artículo 56 refiere "una vez que la Junta Cantonal de Protección de Derechos, conozca sobre la solicitud de otorgamiento de medias administrativas de protección inmediata, verificará por la sola descripción de los hechos, el riesgo de ser vulnerado el derecho a la integridad personal y a la vida digna de las mujeres y las otorgará o denegará inmediatamente" (Ley Orgánica Integral para Prevenir y Erradicar la Violencia Contra las Mujeres, 2018, art. 55).

Del mismo modo, el artículo 11 refiere respecto a los principios de aplicación de los derechos, en su numeral 7 "El reconocimiento de los derechos y garantías establecidos en la Constitución y en los instrumentos internacionales de derechos humanos, no excluirá los demás derechos derivados de la dignidad de las personas, comunidades, pueblos y nacionalidades, que sean necesarios para su pleno desenvolvimiento" (Asamblea Nacional, 2008, art.11).

A este respecto se estaria considerando, que el articulo 11 numeral 7 tiene una estructura que refiere a la dignidad humana; por lo que, ésta razon, es de vital importancia para que la normativa actual pueda reconocer a un futuro respecto a las desiciones que los habitantes de este estado sobre el fin de su vida y estas se darian cuando existan condiciones de salud las cuales indiquen que por su estado deplorable pueden optar por elegir terminar con su vida de forma digna y de acuerdo a su voluntad (Chumbi-Pulla, Erazo-Álvarez, Trelles-Vicuña, \& Narváez-Zurita, 2020). 
Así mismo, el artículo 84 de forma obligatoria dispone que la Asamblea y órganos con potestad jurisdiccional adecuen las leyes a los derechos establecidos en la Constitución, tratados internacionales para que de esta forma se pueda garantizar la dignidad del ser humano (Asamblea Nacional 2008). Por lo que la Asamblea Nacional se encontraría obligada en este caso a regular respecto a la dignidad de las personas en el proceso de muerte, lo cual estaría directamente vinculado con la dignidad de una persona.

Considerando que en la Constitución existen varios artículos que se describen de manera expresa lo que se ha referido anteriormente. El artículo 33 obliga al Estado a garantizar el respeto a la dignidad de los trabajadores. El Articulo 45 hace refiere a una garantía específica para las niñas, niños y adolescentes respeto a su dignidad, y el artículo 57 numeral 21 dentro de los derechos colectivos, reconoce la dignidad de las comunas, comunidades, pueblos y nacionalidades (Asamblea Nacional, 2008).

En general se considera que nuestra constitución, garantiza de manera expresa sobre la dignidad humana se sus habitantes y los grupos que están dentro de estos, pero este tema que es muy importante no se ha valorado de la forma adecuada respecto a la dignidad y decisiones en general de sus habitantes, que en este caso estaría vulnerando esta necesidad sobre la elección de vivir o morir dignamente (Yánez-Chávez, ErazoÁlvarez, Pozo-Cabrera, \& Narváez-Zurita, 2020).

\section{Origen de la Eutanasia y Derecho comparado con legislaciones extranjeras.}

Desde la antigüedad este término fue considerado como un camino a la buena muerte o a la muerte digna solicitada por las personas que les aquejaba una dolencia que prolongaba su agonía y sufrimiento hacia la muerte, es así que este tema tuvo mucha hasta la actualidad. En este contexto Se puede definir a la eutanasia como:

Buena muerte, es decir, la muerte sin dolor, sin sufrimiento. En el siglo XX, durante el Tercer Reich, el término ganó una connotación negativa cuando se utilizó de forma inadecuada en las políticas nazis dirigidas a eliminar vidas que, desde su punto de vista, no valían la pena ser vividas (Parreiras-Reis de Castro, 2016. p.356). 
Luego de analizar varias concepciones de autores respecto a la vida digna y morir dignamente, se analiza varias regulaciones y legislaciones y las concepciones que tienen varios países sobre la eutanasia, por lo tanto, se pretende con esto demostrar sobre lo referente al tema investigado ya que con esto se pretende que esta institución llegue a posicionarse como una garantía en nuestra constitución y así también en pacientes que deseen tener una muerte digna.

En este contexto se analiza lo que refiere la legislación en Estados Unidos de Norteamérica respecto a la muerte digna y eutanasia, en este caso no existe una ley que la ampare y se resuelve por medio de un procedimiento judicial (Molina-Faran, 2008), con la excepción de que en otros estados amparan el suicidio asistido.

Con respecto a Europa y se da por varias resoluciones en el ámbito judicial como se explica a continuación: en Holanda en el año1973, respecto al debate sobre la eutanasia este tuvo su desarrollo en el ámbito judicial a raíz de sucesivas sentencias sobre casos de conductas eutanásicas que, siendo condenatorias, indicaron en sus considerandos las condiciones que, de haberse cumplido en el caso juzgado, habrían dado lugar a una sentencia absolutoria. (Lorda \& Barrio, 2012) "De esta forma fueron los tribunales los que marcaron las circunstancias en las que la acusación de la muerte bajo petición podría ser moral y jurídicamente aceptable"(p.2). Por lo tanto, ya que este término nace de varias resoluciones judiciales las mismas que crearon un precedente en Holanda por lo que se dieron varias circunstancias de muertes bajo petición las cuales se ajustarán a la ley.

La eutanasia a lo largo de los años este tema ha evolucionado de una manera acelerada, pues es cierto que en nuestra legislación no está regulada o no es permitida, pero en países europeos este tema es muy relevante ya que, esta figura jurídica es aceptada, pero la misma puede darse luego de cumplir varios procedimientos y requisitos; en épocas pasadas fue considerada como un delito pues bien ahora no en todos los países europeos está regulada, pero en otros si. Así lo expresa Alonso-Álamo (2008)“la eutanasia activa directa o el suicidio asistido pueden ser tanto prohibidos penalmente como constituir, bajo determinadas circunstancias, excepciones legítimas a la exigencia de protección jurídica de la vida" (p.2). 
Ahora bien, la eutanasia es un procedimiento que puede estar prohibido por la normativa penal en varias legislaciones, pero el mismo sería permitido en ciertas circunstancias, refiriendo a que este tema es importante respecto a la decisión que toma una persona sobre elegir vivir o morir puesto que sus condiciones de vida le causan dolor y sufrimiento, no solo a ellos si no a sus familiares. El modelo del suicidio asistido no se compara en ningún sentido a la eutanasia. En particular, no se resuelven aquellos casos en el que un enfermo se encuentra en fase terminal con sintomatología insoportable; la persona que ha expresado su voluntad de morir no está en condiciones de tomar dicha decisión, en cuanto a casos de personas que se encuentran en estado vegetativo (Alonso-Álamo, 2008).

El problema principalmente es que en el suicidio asistido y la eutanasia es un tema complejo en el que se debe tener compasión de la persona que está pasando por duros momentos de sufrimiento, y al momento que existan garantías que amparen su decisión por morir, así como seguir procedimientos establecidos para el caso, como verificar la voluntad de la persona y estado de raciocinio al momento de tomar esta decisión, por lo tanto existe un grave problema si hay pacientes en estado vegetativo los cuales no pueden decidir respecto a vivir o morir, esta decisión es de sus familiares, pero si se desea aplicar esta normativa, se debe exigir varias condiciones para poder llevar a cabo este procedimiento, como por ejemplo, que esta decisión sea expresa por el paciente o este haya sido su deseo si es que algún día llega a estar en este estado, y así poder garantizar que se ha cumplido con su voluntad. El Código Penal español Textualmente expresa:

El que causare o cooperare activamente con actos necesarios y directos a la muerte de otro, por la petición expresa, seria e inequívoca de éste, en el caso de que la víctima sufriera una enfermedad grave que conduciría necesariamente a su muerte, o que produjera graves padecimientos permanentes y difíciles de soportar, será castigado con la pena inferior en uno o dos grados a las señaladas en los números 2 y 3 de este artículo" (Ministerio de Justicia Español, 1996). 
Estaríamos hablando de una excelente definición de Eutanasia, ya que en la misma se enfoca principalmente a todos los requisitos que deben darse para atenuar penas respecto a conductas tipificadas como homicidio. Ahora bien, en la legislación de Colombia, en el artículo 11 de su Constitución nos dice textualmente que: "El derecho a la vida es inviolable. No habrá pena de muerte" (Corte Constitucional de Colombia, 1991, art.11), si expresamente lo dice esta constitución el derecho a la vida es inviable, y si necesariamente se quisiera incluir en este texto legal lo referente a la eutanasia la forma adecuada seria que: Al no existir una ley expresa en lo referente a peticiones de eutanasia, la forma adecuada seria mediante la interpretación ponderada de principios, valores y derechos (Farfán-Molina, 2008).

En este caso es preciso mencionar que, para aplicar lo referente a la eutanasia en nuestra legislación se podría proceder de la misma forma en la que el autor lo dice, por cuanto realizar una interpretación por parte de la Corte Constitucional respecto a la Eutanasia y así evitar un referéndum o una reforma constitucional, creo que de esta manera seria la forma más adecuada de incluir este derecho.

Con relación al requisito de la voluntad, presenta una exigencia, la persona que se encuentre en estado de salud deplorable, tenga el raciocinio suficiente como para tomar decisiones por sí mismo, por lo que se estaría excluyendo a pacientes que no tengan esta capacidad los cuales estarían sufriendo de alguna patología psiquiátrica la misma le estaría impidiendo de tomar este tipo de decisión la misma que sería importante respecto a su vida por padecer una dolencia que le afecta de manera significativamente.

Entonces debe existir voluntad tácita por parte del solicitante, así como debe existir raciocinio y conciencia por parte del mismo, pero se estaría vulnerando el derecho que los pacientes tienen respecto a decidir si viven o mueren ya que el autor antes citado manifiesta que se podrían excluir pacientes que no tengan capacidad de decidir por ellos mismos, entonces si se consideraría importante incluir en la normativa que regulara la muerte asistida o eutanasia, que se valoren estos casos los mismo que también podrían ser solicitados por sus familiares esto con el fin de evitar más sufrimiento y dolor. 
Respecto a la aplicación de la eutanasia y los abusos que puedan darse al aplicar este procedimiento en pacientes terminales en la tipificación de esta práctica se debe hacer constar características que no lo enmarcan como un delito: no existe norma legal que pueda impedir riesgos en su uso y evitaría abusos en su aplicación; por lo tanto en circunstancias precisas la muerte asistida y la eutanasia no podrían ser punibles, así que si existe alguna ley la misma que tenga como objetivo la despenalización de estas actuaciones debería determinar cuáles deben mantenerse, obviamente, con una calificación de homicidio o de asesinato (Royes, 2008).

En este contexto se dice que no existe norma legal que evite abusos respecto a la aplicación de la eutanasia por lo que esta sería punible si existen circunstancias precisas sobre la aplicación de estos procedimientos médicos por lo cual debe establecerse estas actuaciones y determinarlas por lo cual se estaría enmarcando en un delito o podría ser configurado como asesinato $u$ homicidio.

Para ser mas específicos, para la aplicación de un procedimiento de eutanasia se deben cumplir con varios parámetros; por este motivo, Betancourt (2011) afirma "la eutanasia o muerte asistida, justifica el uso de procedimientos aparentemente médicos para darle solución a una decisión personal de abandonar la vida y es por tanto ajena a la esencia de la profesión médica y a la solidaridad humana." (p.263). Por lo tanto, acertadamente se da un concepto valedero para esta decisión que es tomada por un paciente terminal al cual un médico le da una solución para calmar su sufrimiento por su estado de salud estaríamos entonces hablando de solidaridad ante una dolencia de una persona y que su único alivio sería la muerte.

Para tal efecto la aplicación de este tipo de procedimientos existen varios métodos y mecanismos aplicados a la persona que lo solicita: El empleo inadecuado de la tecnología permite a veces prolongar la vida del paciente durante un período de tiempo variable, pero en ocasiones lo que realmente se prolonga es el proceso de morir, con un coste muy elevado en términos de sufrimiento para el paciente, emocional para su familia, para el personal que le atiende y de recursos para la sociedad (Betancourt , 2011, p. 266). 
El Estado no podría oponerse ante una decisión de una persona que no desea continuar con su vida por lo que él mismo está en la potestad de solicitar ayuda para morir, por el estado de su enfermedad y por los dolores sufridos, por lo tanto el médico podría resistirse a la aplicación de estos procedimientos por lo que entiende que su paciente es un individuo digno por el hecho de ser un ser humano por lo que esa dignidad de ser humano no se pierde por su capacidad de autodeterminarse (Merchán, 2008). Más aún podríamos referir que el estado no tiene la potestad de oponerse ante una decisión de una persona que por una dolencia, esta decide morir y lo cual es una decisión personalísima por lo tanto no se debería haber intromisión alguna por parte de este ya que es una decisión personalísima de la persona.

Se observa también que en el presente caso podemos hay varias formas de aplicar procedimientos de eutanasia, pues la misma puede ser por petición voluntaria de la persona o por petición directa al médico tratante como se refiere el autor que: La petición del paciente puede canalizarse de dos formas diferentes, detalladamente reguladas en la Ley. Una es mediante la petición directa al médico y otra mediante la Voluntad Anticipada.

Los requisitos sobre el estado de salud que tiene que tener el paciente para acceder a la eutanasia han sido motivo de discusión, en particular los referidos a la evaluación del sufrimiento psíquico y a la situación de no terminalidad" (Lorda \& Barrio, 2012, p.12).

Es entonces que primero debe existir valoraciones respecto al estado de salud del paciente, en el ámbito psíquico y estado mental del mismo al momento de tomar una decisión como dice el autor esto ha sido muy cuestionado por cuanto este procedimiento puede acarrear varios problemas jurídicos.

Existen varias formas o clases de eutanasia es por eso que Diez-Ripolles (1995) propone: "clasificación de los supuestos eutanásicos en tres grandes grupos, que denominaría eutanasia terminal, eutanasia paliativa y eutanasia cualitativa, que se estructuran combinando determinadas situaciones clínicas desfavorables con la secuencia temporal por medio de la cual se produce la muerte" (p.89). Por lo tanto 
y desprendiendo de esta clasificación son medios diferentes utilizados para llegar a un mismo fin que sería la muerte digna de la persona que ha solicitado su muerte por medio de este procedimiento.

Al darnos cuenta que existe una clasificación respecto como lo manifiesta el autor podríamos definir estos conceptos respecto a la Eutanasia terminal, paliativa y cualitativa de la siguiente manera: La eutanasia terminal es la que refiere a pacientes terminales y en estado vegetativo. La eutanasia paliativa es la que refiere a las personas incapacitadas e incapaces de valerse por sí mismas por alguna afección incurable o permanente, así que el paciente requiere de medicamentos para mitigar sus dolencias y se le estaría encaminando o preparando para la muerte, y; La eutanasia cualitativa refiere a que se eliminan los padecimientos $o$ dolores sufridos con la provocación de la muerte como único medio.

\section{MÉTODO}

Partiendo del objetivo planteado, el presente estudio fue de tipo no experimental, es decir; la variable independiente puede variar intencionalmente, así como, también pueden analizar fenómenos manifestados de forma natural. El diseño del presente trabajo fue de tipo mixto; cualitativo-cuantitativo, enfocado en recopilación y análisis de artículos científicos en revistas y bases de datos digitales. Cualitativo debido a lo planteado por; Hernández-Sampieri, Fernández-Collado \& Baptista-Lucio (2010) “Utiliza la recolección de datos sin medición numérica para descubrir o afinar preguntas de investigación en el proceso de interpretación" (p.7).

Cuantitativo ya que, (Hernández-Sampieri, Fernández-Collado \& Baptista-Lucio (2010) expresan "En el enfoque cuantitativo los planteamientos a investigar son específicos y delimitados desde el inicio de un estudio y debe ser lo más "objetivo" posible, evitando que afecten las tendencias del investigador u otras personas" (p. 20). Por otro lado, los enfoques utilizados fueron; el inductivo y deductivo, el primero refiere a las conclusiones generales y el segundo a asumir y deducir conclusiones lógicas, al mismo tiempo, es un estudio de método-investigación de tipo histórico-lógico; este método tiene como objetivo 
producir cambios significativos en la realidad analizada, se preocupa por hallar soluciones a problemas específicos a través de la aplicación de una metodología minuciosa. El fin de este método es colocarse en un contexto espaciotemporal, estrechamente unido a la realidad que se inicia desde experiencias reales desde el pasado hacia la actualidad (Behar citado por Abreu 2014, p. 2001). En cuanto al alcance, el estudio fue descriptivo en función de lo planteado por (Pineda y Alvarado, 2008, p. 82).

\section{Universo de estudio y tratamiento muestral}

Con respecto al universo del estudio, fueron encuestados 11 abogados especialistas en materia constitucional a través de la plataforma "Google Forms". Por lo que se refiere, al tratamiento muestral el tipo de muestreo fue no probabilístico de tipo propositivo o por conveniencia debido a que los participantes fueron seleccionados dada la conveniente accesibilidad y disponibilidad.

\section{Tratamiento estadístico de la información}

En relación con el análisis estadístico, los datos se obtuvieron mediante una encuesta la cual fue realizada en "Google Forms", los mismos fueron procesados y analizados, luego tabulados formando así gráficos explicativos a través del programa Microsoft Excel versión 2019 (Erazo-Álvarez \& Narváez-Zurita, 2020).

\section{RESULTADOS}

A través de la aplicación de una encuesta en "Google Forms", los datos que caracterizaron la muestra, se resumen en la Tabla 1 y Figura 1.

\section{Tabla 1}

Resultados de la encuesta aplicada a abogado especialistas en materia Constitucional

\begin{tabular}{lcr} 
& \multicolumn{2}{c}{ Resultado \% } \\
Variable & Si & No \\
\hline Reconocer un derecho & & \\
& $81,80 \%$ & $18,20 \%$
\end{tabular}


Método o forma

Manual sobre eutanasia

Aplicación de la eutanasia

Decisión
$90,90 \%$

$90,90 \%$

$66,60 \%$

$90,90 \%$

$100,00 \%$
Derecho a tomar decisiones

Fuente: Investigadores, 2020.

\section{Resultados}

Se estaria cumpliendo el derecho a la libertad de decisión del paciente

Quién considera usted que debería tomar esta decisión para realizar la autanasia

La eutanasia debe ser aplicada solo para enfermos terminales o para adultos

Se debería incluir un manual o una guia para el paciente, familia y personal de salud

La Eutanasia es un método que podría calmar el sufrimiento de pacientes

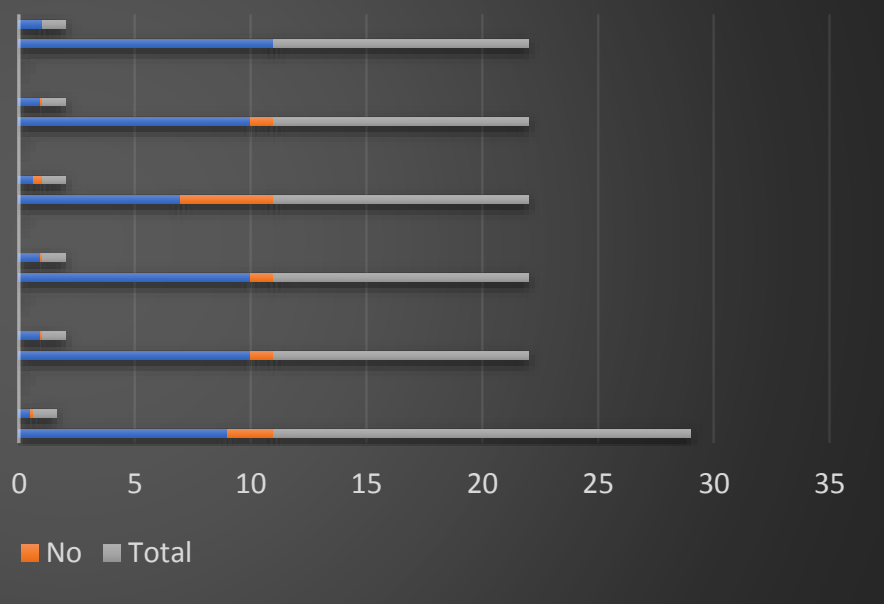

Figura 1 Representación gráfica de los resultados. Fuente: Investigación de campo.

En los resultados se puede evidenciar que, la mayoría de los encuestados manifestaron respecto a cuál sería la forma correcta para que la eutanasia se convierta en un derecho reconocido en la constitución, los mismos en su gran mayoría respondieron 
positivamente así como también manifestaron que la forma correcta para que sea reconocido sea mediante una reforma Constitucional o Referéndum.

En cuanto a los derechos de libertad, estipulados en nuestra Carta magna en el art. 66 el paciente estaría cumpliendo su derecho a la libertad de decisión, por lo tanto los encuestados estarían de acuerdo al responder positivamente respecto a lo preguntado. Así mismo, se ha respondido por parte de los profesionales del derecho en su mayoría que necesariamente se debería incluir un manual o protocolo para la práctica de la eutanasia, por lo tanto, denota que existe una manera correcta de cumplir con los parámetros correctos respecto a lo legal y en lo referente a salud para cumplir con una práctica eutanásica.

En este contexto, se puede concluir que mediante la encuesta realizada, en nuestra constitución no existe un derecho que expresamente garanticé a pacientes terminales, así como la necesidad de que este derecho sea establecido mediante referéndum; $y$, un manual el mismo que garanticé la aplicación de esta práctica la misma que debería constar como un manual en el Ministerio de Salud y de esta forma se estaría garantizando los derechos de libertad y vida digna de los pacientes y enfermos terminales.

\section{PROPUESTA}

Habiendo observado los resultados de las encuestas y el análisis a varios estudios científicos, así como de revistas científicas y derecho comparado con varias legislaciones de otros países, se debería aplicar en el Ecuador este derecho mediante una interpretación evolutiva por parte de la corte Constitucional, ya que es obligación de un estado velar por los derechos de sus habitantes durante la vida así como también en una etapa final de su vida como es la muerte. En esta reforma constitucional respecto a los derechos se incluirá:

\section{1.- Determinar que el derecho a la muerte digna se encuentra implícito en el}

Ecuador: El derecho a una vida digna se encuentra establecido en nuestra Constitución, por lo tanto el estado está obligado a garantizar el mismo y su aplicación 
sin discriminación a ninguno de habitantes y de no cumplirse se estaría vulnerando este derecho. Si nuestra constitución reconoce el derecho a una vida diga, está en la obligación de reconocer el derecho a morir dignamente, por cuanto el Estado la garantizará y de manera adecuada como se ha demostrado que varias legislaciones las mismas que han reconocido el derecho a morir dignamente.

\section{2.- La Corte Constitucional utilizando la interpretación evolutiva deberá insertar el derecho a la muerte digna y eutanasia en la legislación Ecuatoriana: La Corte Constitucional del Ecuador, utilizó esta interpretación y la aplicó al momento de resolver respecto al matrimonio igualitario, con este antecedente se dará dar paso al reconocimiento de una muerte digna.}

\section{3.-El ministerio de Salud, por orden de la Corte Constitucional deberá establecer} un protocolo para la realización de la eutanasia: La Corte Constitucional del Ecuador al ser el máximo órgano de control, interpretación de justicia Constitucional y al haber realizado una interpretación evolutiva respecto al reconocimiento de la muerte digna y eutanasia en el Ecuador, este órgano dispondrá la Ministerio de Salud la realización de un protocolo respecto a los procedimientos y parámetros aplicables para la práctica de la eutanasia, así como responsabilidades y todos los lineamientos a cumplir por parte del paciente, familia y equipo médico. 
Figura 2 Interpretación y reconocimiento del derecho de Eutanasia. Fuente: Elaboración propia

Por lo tanto, la propuesta en esta investigación se ha centrado en la vida digna, la misma que está implícita en un ser humano, por lo que se puede decir que el estado estaría garantizando este derecho. Entonces, el momento que una persona ha podido determinar que su vida no goza de dignidad ni de calidad, producto de una enfermedad terminal, la misma que le está causando sufrimiento, tiene derecho a tomar decisiones sobre su condición, y esta debería ser respetada por el estado y la sociedad.

En este contexto, estas acciones se producen con el desarrollo de su libertad lo cual se encuentra vinculado al proyecto de vida de las personas. De esta manera, surge el derecho al libre desarrollo de la personalidad, por un lado estaría limitando la actuación patriarcal de un estado y por otro haciendo efectiva la libertad de decisión de la persona. Así que, de esta manera se puede determinar que en nuestra carta Magna el derecho a una vida digna sería el camino a una muerte digna.

Es por eso que esta iniciativa se encuentra enfocada especialmente en una interpretación evolutiva utilizada por la Corte Constitucional del Ecuador, pues esta corte la utilizó al 
momento de resolver respecto al matrimonio igualitario y aplicó este tipo de interpretación por lo que con este antecedente se deberá aplicar esta interpretación; y por lo tanto una interpretación evolutiva es el mecanismo por el cual, se entiende en su integralidad y permite reconocer derechos, y por más evolutiva que esta sea, no puede considerarse sinónimo de reforma o cambio de la constitución, y a través de esta categoría se estaría persiguiendo la protección de determinadas instituciones constitucionalmente reconocidas frente a la acción legislativa que pueda intentar suprimirlas o desnaturalizarlas existiendo, entonces, por lógica, un núcleo esencial de la institución que la Constitución garantiza, si la interpretación evolutiva de los preceptos constitucionales resulta, entre nosotros, siempre discutible, lo es mucho más cuando se aplica a una garantía institucional.

\section{DISCUSIÓN}

Acorde con lo mencionado a lo largo del estudio, se evidencia claramente que es factible el derecho a una muerte digna o eutanasia dentro de nuestro país, ya que si se ofrecen derechos que amparan a los seres humanos a lo largo de su vida debe existir uno que lo haga en el final de la misma.

Es así pues que debemos para este momento dejar en claro que no es lo mismo matar que dejar morir, debido a que el segundo le permite al ser humano descansar en paz luego de a veces una larga agonía, no se debe hablar de la "eutanasia" si es que el paciente no ha pedido explícitamente que lo dejen morir, destacando que este debe ser cuidado hasta el final y evitar por encima de todo cada una de sus dolencias tanto físicas, emocionales y hasta espirituales.

Del mismo modo, si un enfermo pide al personal de salud que lo dejen morir o no alarguen su vida, tanto médicos como familiares deben aceptar de forma ética que se debe suspender o no aplicar ningún tratamiento que alargase la misma.

De manera que, lo evidenciado en los resultados y lo expresado por la mayoría de encuestados conocedores de las leyes y derechos en el Ecuador, la eutanasia debería ser reconocida como un derecho, existiendo un manual que guíe al enfermo, la familia 
y al personal sanitario, queda claro también que para la mayoría de las personas que respondieron el cuestionario quién debe tomar esta decisión de morir es el paciente y es por ello la insistencia de una muerte digna y una buena calidad de vida en el final de ella.

No obstante, la eutanasia es un tema delicado, que a pesar de haber sido aprobado y ejecutado en varios países alrededor del mundo, aún es un tabú que duele a muchos debido a lo difícil de aceptar la decisión de un ser querido que debe partir, sin embargo, es un derecho que debe ser respetado, apoyado y contemplado por los familiares y por el personal de salud, partiendo desde la ética y dignidad. Así que, la autonomía del enfermo es la justificación moral para la eutanasia y quién más que el que ha sufrido para tomar esa decisión.

En consecuencia, queda claro que los países que han aprobado la eutanasia (Bélgica, Colombia y Luxemburgo) han tenido que pasar por varias pruebas y algunos años para poder volverlo legal al igual que en (Suiza, Alemania, Japón, Canadá y algunos estados de Estados Unidos) es importante en el campo del derecho una muerte digna.

\section{REFERENCIAS CONSULTADAS}

Abreu, J. L. (2014). El Método de la Investigación. [ The Research Method.] Recuperado de: https://url2.cl/xeh7P. International Journal of Good Conscience., 10.

Alonso-Álamo, M. (2008). Sobre la "Eutanasia y Derechos Fundamentales". [ About "Eutanasia and Fundamental Rights".] Recuperado de:https://url2.cl/pMRXD. Ciencia Penal y Criminología, 1-8.

Amaro-Cano, M. (1998). Una Muerte Digna para una Vida Digna. [ A Worthy Death for a Worthy Life ]. Recuperado de: https://url2.cl/vlf8h. Cuadrenos de Bioética, 822-824.

Arévalo-Arévalo, H. P., Erazo-Álvarez, J. C., Ormaza-Ávila, D. A., \& Narváez-Zurita, C. I. (2020). Delimitantes para el ejercicio legítimo de la Democracia en el Estado Ecuatoriano [Delimiters for the legitimate exercise of Democracy in the Ecuadorian State]. Iustitia Socialis, 697-715. doi:http://dx.doi.org/10.35381/racji.v5i1.637 
Walter Ruperto Mogrovejo-Lituma; Juan Carlos Erazo-Álvarez; Cecilia Ivonne Narváez-Zurita; José Luis Vázquez-Calle

Betancourt , G. (2011). Limitación del esfuerzo terapéutico versus eutanasia: una reflexión bioética.[ Limitation of therapeutic effort versus euthanasia: a bioethical reflection.]. Recuperado de: https://url2.cl/RjBMY. Humanidades Médicas, 259273.

Chumbi-Pulla, T. A., Erazo-Álvarez, J. C., Trelles-Vicuña, D. F., \& Narváez-Zurita, C. I. (2020). La reparación integral y su cuantificación en el daño inmaterial y proyecto de vida [Comprehensive reparation and its quantification in non-pecuniary damage and life project]. Iustitia Socialis, 527-546. doi:http://dx.doi.org/10.35381/racji.v5i8.589

Colombia, C. C. (20 de Julio de 1991). Constitución Política de Colombia. [ Political Constitution of Colombia.]. Principios Fundamentales. Bogotá, Bogotá, Colombia: Corte Constitucional Consejo Superior de la Judicatura Centro de Documentación Judicial -CENDOJ Biblioteca Enrique Low Murtra- BELM.

Decesare, D. (2004). Vida Digna. [ Dignified life ]. Recuperado de: https://url2.cl//dQbZ. Colombia Imágenes y Realidades., 15-172.

Desarrollo, S. N. (22 de septiembre de 2017). Secretaría Nacional de Planificación y Desarrollo. Plan Nacional de Desarrollo 2017- 2021-Toda una Vida. Resolución N ${ }^{\circ}$ CNP-003-2017. [National Development Plan 2017-2021-All Life. Resolution No. CNP-003-2017. ]. Quito, Pichincha, Ecuador: Senplades.

Diez-Ripolles, J. (1995). Eutanasia Y derecho. Anuario de filosofía del derecho XII. [ Euthanasia y Right. yearbook of philosophy of law XII. ] Recuperado de: https://url2.cl/4jyHD. Eutanasia y Derecho, 83-114.

Erazo-Álvarez, J. C., \& Narváez-Zurita, C. I. (2020). Medición y gestión del capital intelectual en la industria del cuero - calzado en Ecuador. [Measurement and management of intellectual capital in the leather industry - footwear in Ecuador]. Revista Arbitrada Interdisciplinaria Koinonía, 437-467. doi:http://dx.doi.org/10.35381/r.k.v5i9.662

Escobar-Triana, J. (1998). El morir, como ejercicio final del Derecho a una vida digna. [Dying, as a final exercise of the Right to a decent life ]. Recuperado de: https://url2.cl/T5ICP. Revista Colombiana para los profesionales de la Salud, 1-2.

Farfán-Molina, F. (2008). Eutanasia, Derechos Fundamentales y ley penal. [ Euthanasia, Fundamental Rights and criminal law]. Bogotá: Nueva Jurídica.

Hernández-Sampieri, R. F.-C.-L. (2010). Metodología de la investigación. Quinta edición. [ Investigation methodology. Fifth edition.]. Recuperado de: https://n9.cl/px0c. México: McGraw-Hill / Interamericana Editores, S.A. DE C.V. 
Walter Ruperto Mogrovejo-Lituma; Juan Carlos Erazo-Álvarez; Cecilia Ivonne Narváez-Zurita; José Luis Vázquez-Calle

Justicia, M. d. (25 de mayo de 1996). Código Penal Español. [ Spanish Penal Code.]. Delitos y Penas; del homicido y sus formas. Madrid, Madrid, España: Boe.

Lorda, P., \& Barrio, I. (2012). Eutanasia en Bélgica ¿un modelo para España?. [ Euthanasia in Belgium ¿a model for Spain?] Recuperado de: https://url2.cl/7bf6A. Revista Española de Salúd Pública, 1-4.

Lorda, P., \& Barrio, I. (2012). Eutanasia en Bélgica ¿Un modelo para España?. [ Euthanasia in Belgium. A model for Spain?] Recuperado de: https://url2.cl/7bf6A. Revista Española Salúd Pública, 5-19.

Ludwing-Schmidt, H. (2013). ¿Vida digna o muerte digna? Concepciónes actuales. [Worthy life or worthy death? Current conceptions. ] Recuperado de: https://url2.cl/rv6L9. Revista Bioethikos, 157-173.

Martínez-Sempere, E. (2000). El Derecho a una vida digna. [The right to a decent life until the end: suicide and euthanasia ] Recuperado de: https://url2.cl/9dG6V. Ética $y$ Derecho, 15-24.

Medina-Morales, D. (2013). Muerte digna, vida digna. Una reflexión - Un debate. [ Death worthy life worthy. A reflection - A debate]. Recuperado de: https://url2.cl/P62Um. Cuadernos De Bioetica, 399-416.

Merchán, J. P. (2008). La Eutanasia no es un acto médico. Persona y Bioética. [Euthanasia is not a medical act. Person and Bioethics ]. Recuperado de: https://url2.cl/52tGe. Persona y Bioética, 42-52.

Molina-Faran, F. (2008). Eutanasia, derechos fundamentales y ley penal. [ Euthanasia, fundamental rights and criminal law.]. Bogotá: Nueva Juridica.

Montón, C. (25 de octubre de 2015). Vida Digna, Muerte Digna. [worthy life, worthy death]. El Siglo, págs. 12-15.

Nacional, A. (20 de octubre de 2008). Constitución de la República del Ecuador. [ Constitution of the Republic of Ecuador.]. Derechos. Montecristi, Manabí, Ecuador: Registro oficial 449.

Nacional, A. (20 de Octubre de 2008). Constitución de la República del Ecuador. [ Constitution of the Republic of Ecuador.]. Derechos de Libertad. Montecristi, Manabí, Ecuador: Registro oficial 449.

Nacional, A. (20 de octubre de 2008). Constitución de la República del Ecuador. [ Constitution of the Republic of Ecuador.]. Derechos de Libertad. Montecristi, Manabí, Ecuador: Registro Oficial 449. 
Walter Ruperto Mogrovejo-Lituma; Juan Carlos Erazo-Álvarez; Cecilia Ivonne Narváez-Zurita; José Luis Vázquez-Calle

Nacional, A. (20 de Octubre de 2008). Constitución de la República del Ecuador. [ Constitution of the Republic of Ecuador]. Derechos. Monrecristi, Manabí, Ecuador: Registro oficial 449.

Nacional, A. (20 de Octubre de 2008). Constitución de la República del Ecuador. [ Constitution of the Republic of Ecuador.]. Garantías Constituciónales. Montecristi, Manabí, Ecuador: Registro oficial 449.

Nacional, A. (05 de Febrero de 2018). Ley Orgánica Integral para Prevenir y Erradicar la Violencia Contra las Mujeres. [Comprehensive Organic Law to Prevent and Eradicate Violence Against Women.]. Art. 55. Quito, Pichincha, Ecuador: Registro Oficial 175.

Parreiras-Reis de Castro, C.-A. P.-M.-A. (2016). Eutanasia y suicidio asistido en países occidentales: una revisión sistemática.[Euthanasia and assisted suicide in western countries: a systematic review. ] Recuperado de: https://url2.cl/aPltj. Revista de Bioetica, 355-367.

Pineda, y. A. (2008). Metodología de la investigación. [ Investigation methodology]. Recuperado de: https://url2.cl/LM69V. Washington, DC.: Organización Panamericana de la Salud.

Royes, A. (2008). La Eutanacia y el suicidio Medicamente Asistido. [ Euthanasia and Medically Assisted Suicide.] Recuperado de: https://url2.cl/HeYb8. Psicooncología. Vol. 5, 223-337.

Sarmiento-Medina, V.-C. V.-J. (2012). Problemas y decisiones al final de la vida en pacientes con enfermedad en etapa terminal.[ Problems and decisions at the end of life in patients with end-stage disease.] Recuperado de: https://url2.cl/gjbCq. Revista de Salud Publica, 116-128.

Yánez-Chávez, N. V., Erazo-Álvarez, J. C., Pozo-Cabrera, E. E., \& Narváez-Zurita, C. I. (2020). La regulación jurídica de la donación y trasplantes anatómicos del Ecuador desde una perspectiva constitucional [The legal regulation of donation and anatomical transplants of Ecuador from a constitutional perspective]. Iustitia Socialis, 312-329. doi:http://dx.doi.org/10.35381/racji.v5i1.614

(C2020 por los autores. Este artículo es de acceso abierto y distribuido según los términos y condiciones de la licencia Creative Commons Atribución-NoComercial-Compartirlgual 4.0 Internacional (CC BY-NC-SA 4.0) (https://creativecommons.org/licenses/by-nc-sa/4.0/). 